\title{
ISOLATION, CHARACTERIZATION, AND ANTIMICROBIAL PROFILING OF BACTERIA FOUND ON THIKA LEVEL 5 HOSPITAL SURFACES, KENYA
}

\author{
Raphael Nyaruaba ${ }^{*}$, Caroline $^{\text {Mwaliko }}{ }^{2}$, and Caroline Wangari Ngugi ${ }^{1}$ \\ 1. Department of Medical Microbiology, Jomo Kenyatta University of Agriculture and Technology (JKUAT), \\ Kenya. \\ 2. Department of Botany, Jomo Kenyatta University of Agriculture and Technology (JKUAT), Kenya. \\ *Corresponding author. E-mail: rohurul@gmail.com. Tel: +254704162300/+8618801276765.
}

\section{ABSTRACT}

Multiple studies have shown that hospital settings are poorly cleaned during terminal cleaning. The adequacy of these cleaning methods has been undermined by presence of multi drug resistant bacteria on hospital surfaces. This case is even more serious in developing countries leading to health care- associated infections that pose a great threat to patients, visitors and health care providers in hospital settings.

This study used various microbiological techniques to test for antibiotic susceptibility profiles of bacteria present at Thika Level 5 Hospital surfaces, Kenya. A simple random cross sectional study was performed, with a total of 85 samples being collected from five different sites. The sites included male and female wards, health care personnel offices, latrine, and kitchen surfaces. Samples were collected using sterile swabs, dipped in normal saline, and transported to the laboratory within 2 Hours for processing.

Of the 85 plates cultured, 47 plates showed bacterial growth $(55 \%)$ on selective media with a significant $\mathrm{P}$ value of 0.0357 . Seven different species of bacteria were identified biochemically from all sites, Escherichia coli was the most abundant species (28\%), and the least was Salmonella typhii (5\%). Multiple drug resistance was common in the different bacteria identified. All isolates were resistant to chloramphenical and susceptible to gentamycin. The most resistant microorganism was Staphylococcus aureus (50\%), and the least resistant microorganism was Klebsiella pneumoniae (12.5\%). The antimicrobial resistant bacterial species identified in this study have been documented to cause serious health care associated infections. These results present a significant public health concern because there is a possibility of patients, staff and visitors contacting nosocomial infections when they come into contact with surfaces at Thika Level 5 Hospital surfaces, Kenya.

Key words: Hospital surfaces, antibiotics, identification, bacteria, Thika Level 5 Hospital. 


\section{INTRODUCTION}

The study of bacteriology begun in 1976 when Antonie van Leeuwenhoek discovered small living creatures microscopically (1632-1723), and communicated his findings with Robert Hooke (16351703) [1]. The spread of diseases like syphilis prompted the development of antibiotics, including Salvarsan which may be among the first antibiotics to be produced [2]. From then till now a number of antimicrobials have been developed especially with the emergence of antibiotic resistant bacteria [2].

Several studies have provided evidence of the existence of antimicrobial agents on environmental surfaces. This case is more severe when it comes to hospital surfaces [3-5]. Contamination of hospital environmental surfaces including porous (like curtains and bed sheets), and non-porous hard surfaces (like tables and charts) may be due to a variety of factors such as dispersion that leads to widespread environmental contamination, enhanced strain survival, and relative resistance to disinfectants used on environmental surfaces [4,6]. Ensuring a healthy environment during hospitalization has been said to be critical in combating hospital acquired infections [5].

Cleaning of hospital surfaces has been the key step geared towards reducing hospital careassociated infections (HAIs), but studies show that terminal cleaning techniques are not that effective in decontaminating hospital environmental surfaces $[3,5,6]$. This is even serious with the emergence of environmentally resistant microorganisms like methicillin-resistant Staphylococcus aureus (MRSA), vancomycin-resistant Enterococcus (VRE), Clostridium difficile, Acinetobaacter, and norovirus [4].

Developing countries have been shown to have endemic HAIs that present a major burden and public health safety issue when it comes to patient, visitors and health care providers safety in hospital environments [7]. Kenya is one of the developing countries in the world and has been documented to show antimicrobial resistance [8]. This study will focus on a single hospital in Kenya to isolate, characterize and identify medically significant pathogens in hospital environmental surfaces that show resistance to antibiotics. 


\section{METHODS}

\section{Study site}

Thika Level 5 Hospital is a governmental hospital with a bed capacity of 300 and seven inpatient wards. The hospital is located 50 kilometers north east of Nairobi town, which is in the central province of Kenya [9]. This hospital provides pediatric, surgical, medical, gynecological, and obstetric services to a mixed rural to urban population [10].

\section{Study design and sample size}

The study design was a cross sectional hospital based study and samples were randomly collected from different sites in the hospital. A total of 85 samples were collected for this study due to financial constraints.

\section{Number of bacterial samples collected per surface}

The sample sites or location were grouped into five major regions. These regions included region 1 which was the male ward, region 2 which was the female ward, region 3 which was the healthcare personnel offices, region 4 which was the kitchen, and region 5 which was the latrine surfaces. A total number of 17 samples were collected randomly from every site. The surfaces to be sampled randomly in these sites included a variety of keyboards, kitchen surfaces, hospital trays, crash carts, charts, medical records, and a patient's entire bedside area which also includes the patient's bedside table, chair, frame etc.

\section{Sample collection}

Sterile cotton wool swab sticks wetted by dipping in normal saline were used to collect samples from hospital surfaces. Samples were collected by swabbing different surfaces in the five sites including non-porous hard surfaces (example like chairs and tables), and porous surfaces (examples like curtains and bed sheets). The swabs were stored in sterile tubes, tightly capped and labelled appropriately ready for transportation in an ice cool box to the Jomo Kenyatta University of Agriculture and Technology (JKUAT) Medical Microbiology Laboratory for processing.

\section{Sample inoculation}

The swab sticks were each used within 2 hours after collection to inoculate plates of Eosin Methylene Blue (EMB), Mannitol Salt Agar (MSA), Deoxycholate Citrate Agar (DCA), MacConkey, and Cysteine Lactose Electrolyte-deficient (CLED) according to the manufacturer's instructions [11]. The plates were incubated for $24 \mathrm{~h}$ at $37^{\circ} \mathrm{C}$. Bacterial colonies that grew on these media were sub cultured on nutrient agar. All isolates that grew as pure cultures were tested biochemically. 


\section{Sample identification}

Bacteria was identified based on the isolates morphological and biochemical characteristics. Morphological characteristics observed after $24 \mathrm{~h}$ growth were based on colony appearance, size, color, shape, elevation, and gram stain. Biochemical tests were done for identification of suspect organisms. The biochemical tests performed were starch, indole [12], urease, catalase [13], VogesProskaeur (VP) [14], gelatin broth, citrate utilization, and methyl red tests according to the manufacturer's instructions. The test results were recorded to be either positive or negative.

\section{Antimicrobial susceptibility testing}

Suspected organisms identified biochemically were tested for their antibiotic susceptibility profiles using commercially purchased antibiotic discs. The zones of inhibition were measured in $\mathrm{mm}$ and rounded off to the nearest whole number to classify bacteria to be either susceptible, intermediate or resistant based on CLSI 2010. Antibiotics used for this study were Ampicillin $(25 \mu \mathrm{g})$, Tetracycline $(25 \mu \mathrm{g})$, Clotrimazole $(25 \mu \mathrm{g})$, Streptomycin $(10 \mu \mathrm{g})$, Kanamycin $(30 \mu \mathrm{g})$, Gentamicin $(10 \mu \mathrm{g})$, Sulphamethaxozole $(200 \mu \mathrm{g})$, and Chloramphenicol (30 $\mu \mathrm{g})$.

Data analysis and presentation was done using Microsoft Excel and GraphPad prism software. 


\section{RESULTS}

Of the 85 plates cultured, 47 (55\%) plates grew bacteria. Most growth was observed in MSA (34\%), followed by CLED (30\%), EMB (26\%), MacConkey (6\%), and DCA (4\%). The growth of bacteria on the different media was significant with a $\mathrm{P}$ value of $\mathrm{P}=0.0357$ calculated by a two way ANOVA based on GraphPad prism software as shown in figure 1.Of the total 17 samples swabbed from different sites, most bacterial growth occurred in kitchen site surfaces $(82 \%)$, followed by latrine site surfaces (53\%), and the rest of the three sites showed equal bacterial growth $(47 \%)$.

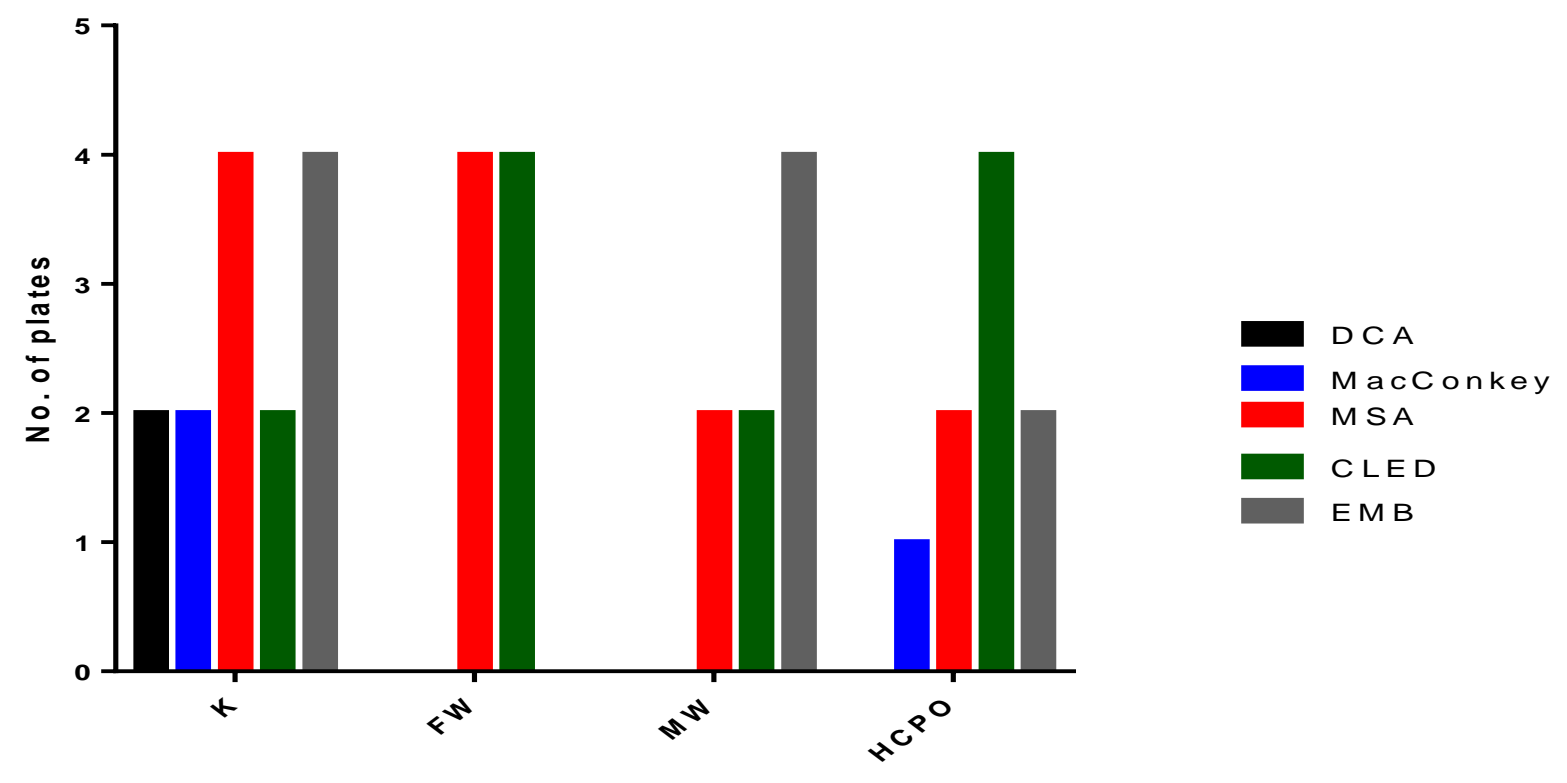

Figure 1. Number of bacterial growth on various media per sampling area.

K-Kitchen, FW- female ward, MW-Male ward, HCPO-health care personnel office, L-latrine

Four representative samples per site that grew on culture were subjected to biochemical tests for identification. Seven different bacteria were identified biochemically with the highest isolate being Escherichia coli (28\%), and the lowest being Salmonella typhii (5\%) as presented in figure 2. E. coli was isolated from all surfaces except latrine surfaces, which accounts for its high prevalence. Proteus vulgaris and Enterobacter aerogenes were isolated from single sites which include latrine 
and male ward sites respectively. Four of the seven bacteria identified biochemically were seen on Kitchen surfaces, hence showing the highest diversity among the five different sites.

\section{Total number of bacteria identified biochemically}

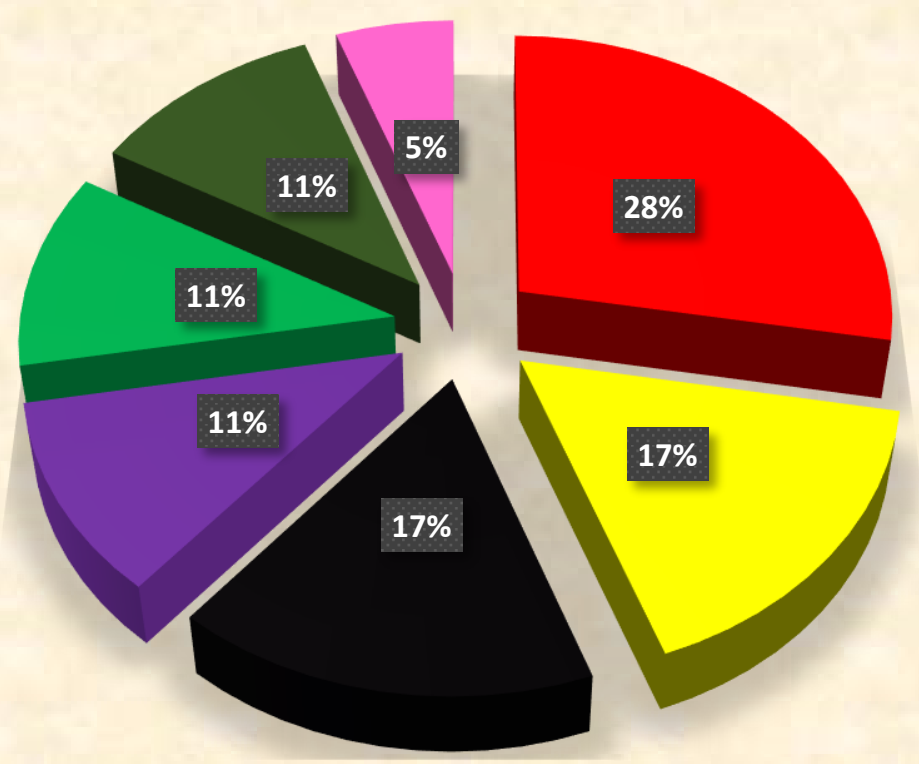

- E coli

Saureus

- $P$ aureginosa

K pneumoniae

- $P$ vulgaris

E aerogenes

S typhii

Figure 2: Total number of bacteria identified biochemically

Multiple drug resistance was common in different bacteria isolated from hospital surfaces as shown in figure 3. All isolates were resistant to Chloramphenical and susceptible to gentamycin. The most resistant microorganism was $S$. aureus $(50 \%)$, and the least resistant microorganism was Klebsiella pneumoniae (12.5\%). Pseudomonas aeruginosa showed resistance to 3 antibiotics and intermediate to two antibiotics making it the least susceptible microorganism in the hospital surfaces. 


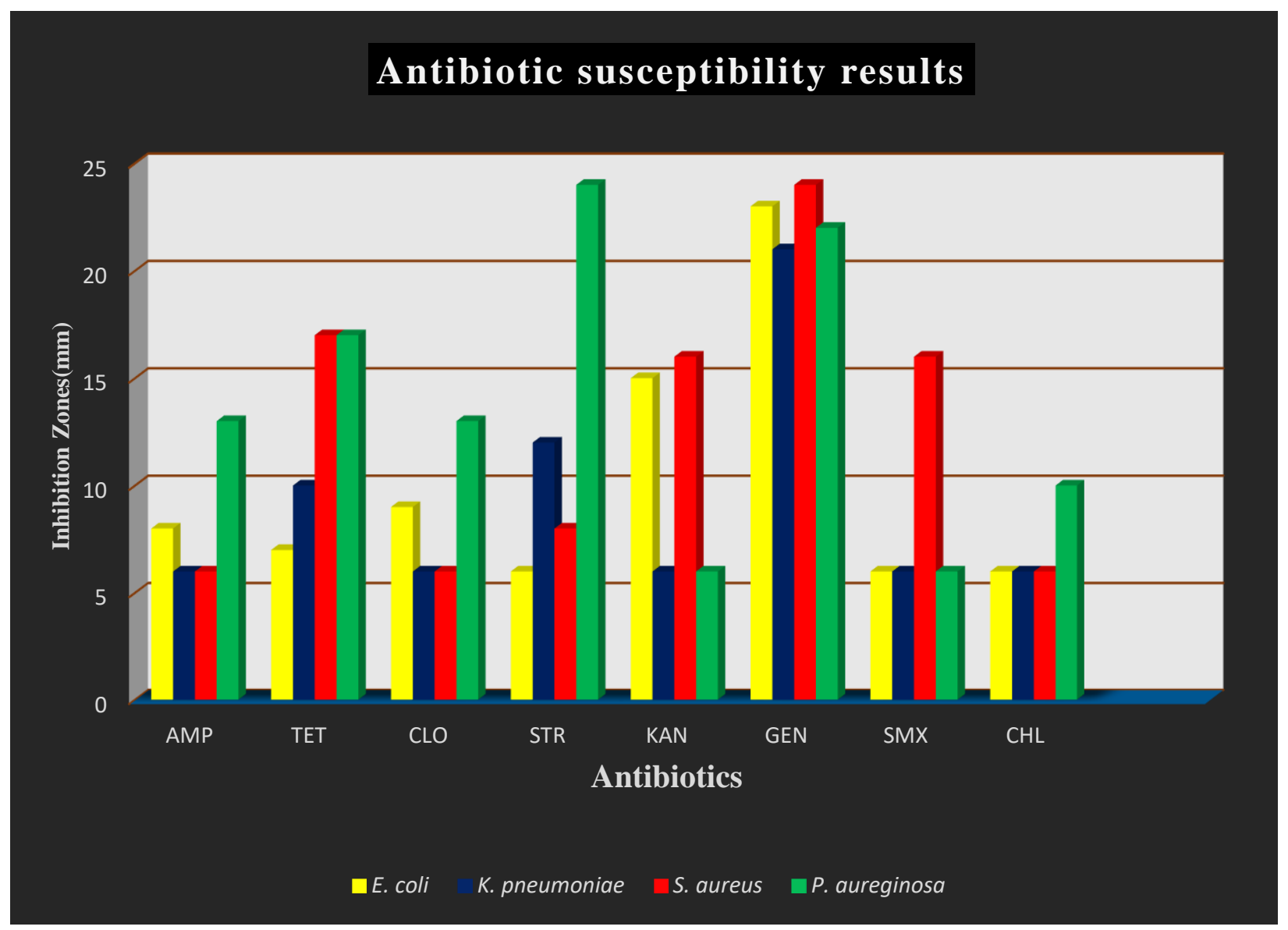

Figure3: Antibiotic susceptibility results

AMP- Ampicillin, TET- Tetracycline, CLO- Clotrimazole, STR-Streptomycin, KANKanamycin, GEN- Gentamycin, SMX-Sulphamethaxozole, CHL- Chloramphenical. 


\section{DISCUSSION}

This study aimed at isolating, characterizing and establishing the antimicrobial profiles of various bacteria present on hospital surfaces. Growth of bacteria observed on culture and subculture provided a basis for the subsequent characterization using various biochemical tests, and antibiotic susceptibility to test resistance.

Bacteria that were isolated from various surfaces grew significantly $(\mathrm{P}=0.0357)$ on different media as shown in figure $1.55 \%$ of the total plates grew different bacterial isolates, this is almost similar to a study done previously [11]. The biggest percentage of bacterial growth was observed on MSA (34\%), followed by CLED (30\%), EMB (26\%), MacConkey (6\%), and DCA (4\%). An evaluation on growth observed on various surfaces shows that; kitchen surfaces were the most contaminated occupying $30 \%$ of the total number of plates that grew. Contamination of kitchen surfaces can be from various sources including humans and food. Just like in this study, Salmonella and other bacteria species have been shown to be isolated from kitchen surfaces including chopping boards [15]. Female and male wards showed growth only on MSA, CLED, and EMB. This is the same case with the health care personnel office isolates. Latrine surface isolates showed growth in four of the five media cultured. It was the second highest contaminated surface after the kitchen surfaces. The significant growth observed provides evidence of contamination on various site surfaces in Thika Level 5 Hospital surfaces.

Out of the 19 bacterial isolates tested biochemically, only 7 suspected bacterial organisms were identified (Figure 2) with $E$. coli being the most abundant species $(28 \%)$, and $S$. typhii being the least abundant species (5\%). Previous studies have also shown that different bacteria can be isolated from environmental surfaces with different prevalence [16]. Bacteria being located in hospital surfaces has been linked with HAIs, and this presents a great challenge to both the clinicians and health care officers [3,4]. Weber and his colleagues also listed factors that can facilitate surface environment-mediated transmission of selected pathogens. Here they highlighted that pathogens can survive in environmental surfaces for a very long time, and these pathogens can remain harmful in the environment especially in hospital surfaces [4]. In this study, hospital surface environmental contamination could also be attributed to the fact that large number of patients visit this hospital. Since Thika level 5 Hospital is a public hospital, many patients prefer services here due to its affordability. This poses a potential threat of cross contamination from patients, visitors and workers in this hospital who are in constant contact with hospital environmental surfaces. This case has also been highlighted by a study done in various hospitals in Kenya [16]. A review done by Russotto and his colleagues highlighted that $20-40 \%$ of nosocomial infections arise from cross contamination from healthcare personnel hands. They also stated that inanimate surfaces in a patients zones are directly contaminated when patients shed bacteria, or indirectly due to the constant interactions with heath care workers hands and touching surfaces, in the patient zones [17].

Multi drug resistant bacteria were found to be present in Thika Level 5 Hospital surfaces as shown in Figure 3. Gentamicin was the most effective antibiotic due to all bacteria being susceptible to this antibiotic. Gentamicin has been shown to be an effective antibiotic against various bacteria 
and potential applications of this antibiotic against gram positive and gram negative bacteria are being explored [18,19]. Chloramphenicol was the worst performing antibiotic with all bacteria showing resistance to this antibiotic. Chloramphenicol resistance has been studied and mechanisms that confer resistance to this antibiotic have been established. These mechanisms include some bacteria like $E$. coli being able to inhibit and inactivate the antibiotics activity completely [20]. E. coli has been studied before and this bacteria has been documented to show resistance to tetracycline and a wide variety of antibiotics [21]. In this study E. coli was resistant to only two antibiotics gentamicin and kanamycin. S. aures isolated in this study was resistant to four $(50 \%)$ out of the 8 antibiotics tested. Previous studies have shown the presence of antibiotic resistant S.aureus in many environmental surfaces including hospitals, especially methicillinresistant Staphylococcus aureus [3,6]. A study done in Thika Level 5 Hospital in 2011 showed the presence of drug resistant $S$.aureus isolated from patients nasal swabs attending this hospital [9]. These patients may spread this bacteria to environmental surfaces through cross contamination posing a risk factor to fellow patients, workers, and visitors. $K$. pneumonia was resistant only to a single antibiotic (gentamicin). Contrary to this study, extended-spectrum $\beta$-lactamase producing strains of Klebsiella pneumoniae have been shown to cause resistance to many antibiotics [22,23]. $P$. aeruginosa showed resistance to 3 antibiotics and intermediate to two antibiotics making it the least susceptible microorganism in the hospital surfaces. This bacterial species has showed varying susceptibility and resistance patterns to antibiotics in other studies [24]. Resistance of bacteria to the various antibiotics could be attributed to various facts e.g. biofilm formation, genetic composition and production of various enzymes capable of inhibiting antibiotic activities on the bacterial cell [25].

These results present a significant public health concern because there is a possibility of patients, staff and visitors contacting HAIs when they come into contact with these surfaces. The burden of contacting HAIs especially in developing countries is high. This is even elevated with inadequate hygienic conditions, understaffing, poor infrastructure, overcrowding and many other factors that are seen in resource limited hospitals [7].

\section{Conclusion}

This work provides evidence that different bacterial species exist in the hospital environmental surfaces. Isolation of these potentially pathogenic bacteria from hospital surfaces suggests that people visiting hospitals should exercise proper hygienic techniques including hand wash, use of disinfectants and/or sanitizers, and where possible use of gloves when in contact with hospital surfaces. This will help reduce the burden of nosocomial infection. The presence on antibiotic resistant bacteria suggests that more effective antimicrobials should be used during terminal cleaning and decontamination of hospital surfaces.

\section{Acknowledgement}

The authors thank Jomo Kenyatta University of Agriculture and Technology (JKUAT) for funding this work. We also thank the staff and students of JKUAT Medical Microbiology for their contribution towards the successful completion of this project 


\section{REFERENCES}

[1] Gould K. Antibiotics: From prehistory to the present day. J Antimicrob Chemother 2016;71:5725. doi:10.1093/jac/dkv484.

[2] Saga T, Yamaguchi K. History of antimicrobial agents and resistant bacteria. Japan Med Assoc J 2009;52:103-8. doi:10.1016/S2222-1808(13)60066-3.

[3] Boyce JM. Environmental contamination makes an important contribution to hospital infection. J Hosp Infect 2007;65:50-4. doi:10.1016/S0195-6701(07)60015-2.

[4] Weber DJ, Rutala WA, Miller MB, Huslage K, Sickbert-Bennett E. Role of hospital surfaces in the transmission of emerging health care-associated pathogens: Norovirus, Clostridium difficile, and Acinetobacter species. Am J Infect Control 2010;38:S25-33. doi:10.1016/j.ajic.2010.04.196.

[5] Caselli E. Hygiene: microbial strategies to reduce pathogens and drug resistance in clinical settings. Microb Biotechnol 2017;10:1079-83. doi:10.1111/1751-7915.12755.

[6] Wagenvoort JHT, Sluijsmans W, Penders RJR. Better environmental survival of outbreak vs. sporadic MRSA isolates. J Hosp Infect 2000;45:231-4. doi:10.1053/jhin.2000.0757.

[7] Allegranzi B, Nejad SB, Combescure C, Graafmans W, Attar H, Donaldson L, et al. Burden of endemic health-care-associated infection in developing countries: Systematic review and metaanalysis. Lancet 2011;377:228-41. doi:10.1016/S0140-6736(10)61458-4.

[8] Omulo S, Thumbi SM, Njenga MK, Call DR. A review of 40 years of enteric antimicrobial resistance research in Eastern Africa: What can be done better? Antimicrob Resist Infect Control 2015;4:113. doi:10.1186/s13756-014-0041-4.

[9] Aiken AM, Wanyoro AK, Mwangi J, Mulingwa P, Wanjohi J, Njoroge J, et al. Evaluation of surveillance for surgical site infections in Thika Hospital, Kenya. J Hosp Infect 2013;83:140-5. doi:10.1016/j.jhin.2012.11.003.

[10] Aiken AM, Mutuku IM, Sabat AJ, Akkerboom V, Mwangi J, Scott JAG, et al. Carriage of Staphylococcus aureus in Thika Level 5 Hospital, Kenya: A cross-sectional study. Antimicrob Resist Infect Control 2014;3:1-7. doi:10.1186/2047-2994-3-22.

[11] Orji MU, Mbata TI, Kalu OU. Isolation of pathogenic bacteria from hospital staff apparel in Nigeria. Malawi Med J 2005;17:128-30. doi:10.1111/j.1365-2516.2007.01459.x.

[12] Abiola C, Oyetayo VO. Isolation and Biochemical Characterization of Microorganisms Associated with the Fermentation of Kersting's Groundnut (Macrotyloma geocarpum). Res J Microbiol 2016;11:47-55. doi:10.3923/jm.2016.47.55.

[13] Cheesbrough M. District laboratory practice in tropical countries. Cambridge university press; 2006.

[14] Sylvia M. Methyl Red and Voges-Proskauer Test Protocols. 2009.

[15] Zhao P, Zhao T, Doyle MP, Rubino JR, Meng J. Development of a Model for Evaluation of Microbial Cross-Contamination in the Kitchen. J Food Prot 1998;61:960-3. doi:10.4315/0362028X-61.8.960. 
[16] Maina SM, Nyerere AK, Ngugi CW. Isolation of bacterial diversity present in medical waste and health care settings in hospitals in Kenya. African J Microbiol Res 2018;12:606-15.

[17] Russotto V, Cortegiani A, Raineri SM, Giarratano A. Bacterial contamination of inanimate surfaces and equipment in the intensive care unit. J Intensive Care 2015;3:54. doi:10.1186/s40560-0150120-5.

[18] Aelenei P, Rimbu CM, Guguianu E, Dimitriu G, Aprotosoaie AC, Brebu M, et al. Coriander essential oil and linalool - interactions with antibiotics against Gram-positive and Gram-negative bacteria. Lett Appl Microbiol 2018:0-1. doi:10.1111/lam.13100.

[19] Mullins ND, Deadman BJ, Moynihan HA, McCarthy FO, Lawrence SE, Thompson J, et al. The impact of storage conditions upon gentamicin coated antimicrobial implants. J Pharm Anal 2016;6:374-81. doi:10.1016/j.jpha.2016.05.002.

[20] SHAW W V. BACTERIAL RESISTANCE TO CHLORAMPHENICOL. Br Med Bull 1984;40:36-41. doi:https://doi.org/10.1093/oxfordjournals.bmb.a071945.

[21] Kibret M, Abera B. Antimicrobial susceptibility patterns of E. coli from clinical sources in northeast Ethiopia. Afr Health Sci 2011;11. doi:10.4314/ahs.v11i3.70069.

[22] Feizabadi MM, Etemadi G, Yadegarinia D, Rahmati M, Shabanpoor S, Bokaei S. Antibioticresistance patterns and frequency of extended-spectrum beta-lactamase-producing isolates of Klebsiella pneumoniae in Tehran. Med Sci Monit 2006;12:BR362-5. doi:9175 [pii].

[23] Lin WP, Wang JT, Chang SC, Chang FY, Fung CP, Chuang YC, et al. The Antimicrobial Susceptibility of Klebsiella pneumoniae from Community Settings in Taiwan, a Trend Analysis. Sci Rep 2016;6:1-11. doi:10.1038/srep36280.

[24] Sader HS, Huband MD, Castanheira M, Flamm RK. Antimicrobial Susceptibility Results from Four Years (2012 to 2015) of the International Network for Optimal Resistance Monitoring Program in the United States. Antimicrob Agents Chemother 2017;61.

[25] Munita JM, Arias CA. Mechanisms of Antibiotic Resistance. Microbiol Spectr 2016;4:10.1128/microbiolspec.VMBF-0016-2015. doi:10.1128/microbiolspec.VMBF-0016-2015. 